\title{
Quantitative Profiling of Oxylipins in Acute Experimental Intracerebral Hemorrhage
}

\begin{abstract}
Jun-Jie Yuan, Qiong Chen, Xiao-Yi Xiong, Qin Zhang, Qi Xie, Jia-Cheng Huang, Guo-Qiang Yang, Chang-Xiong Gong, Zhong-Ming Qiu, Hong-Fei Sang, Wen-Jie Zi, Qian He, Rui Xu and Qing-Wu Yang*
\end{abstract}

Department of Neurology, Xinqiao Hospital, The Army Medical University (Third Military Medical University), Chongqing, China

Oxylipins are a series of bioactive lipid metabolites derived from polyunsaturated fatty acids that are involved in cerebral homeostasis and the development of intracerebral hemorrhage $(\mathrm{ICH})$. However, comprehensive quantification of the oxylipin profile in $\mathrm{ICH}$ remains unknown. Therefore, an $\mathrm{ICH}$ mouse model was constructed and liquid chromatography tandem mass spectrometry was then performed to quantify the change in oxylipins in $\mathrm{ICH}$. The expression of the oxylipin relative enzymes was also reanalyzed based on RNA-seq data from our constructed ICH dataset. A total of 58 oxylipins were quantifiable and the levels of 17 oxylipins increased while none decreased significantly in the first 3 days following $\mathrm{ICH}$. The most commonly increased oxylipins in $\mathrm{ICH}$ were derived from AA (10/17) and EPA (4/17) followed by LA (2/17) and DHA (1/17). 18-HEPE from EPA was the only oxylipin that remained significantly increased from 0.5 to 3 days following $\mathrm{ICH}$. Furthermore, 14 of the increased oxylipins reached a peak level on the first day of $\mathrm{ICH}$, and soon decreased while five oxylipins (PGJ2, 15-oxo-ETE, 12HEPE, 18-HEPE, and 5-Oxo-ETE) had increased 3 days after ICH suggesting that the profile shifted with the progression of $\mathrm{ICH}$. In our constructed RNA-seq dataset based on $\mathrm{ICH}$ rats, 90 oxylipin relative molecules were detected except for COX. Among these, Cyp4118, Cyp1b1, Cyp2d3, Cyp2e1, Cyp1a1, ALOX5AP, and PLA2g4a were found up-regulated and Cyp26b1 was found to decrease in $\mathrm{ICH}$. In addition, there was no significant change in $s E H$ in $\mathrm{ICH}$. This study provides fundamental data on the profile of oxylipins and their enzymes in $\mathrm{ICH}$. We found that the profile shifted as the progression of $\mathrm{ICH}$ and the metabolism of arachidonic acid and eicosapentaenoic acid was highly affected in $\mathrm{ICH}$, which will help further studies explore the functions of oxylipins in $\mathrm{ICH}$.

Accepted: 02 July 2020

Published: 23 September 2020

Citation:

Yuan J-J, Chen $Q$, Xiong X-Y,

Zhang $Q$, Xie $Q$, Huang J-C,

Yang G-Q, Gong C-X, Qiu Z-M,

Sang H-F, Zi W-J, He Q, Xu R and

Yang Q-W (2020) Quantitative

Profiling of Oxylipins in Acute

Experimental Intracerebral

Hemorrhage.

Front. Neurosci. 14:777.

doi: 10.3389/fnins.2020.00777
Keywords: oxylipin, intracerebral hemorrhage, cyclooxygenase, lipoxygenase, cytochrome P450

Abbreviations: AA, arachidonic acid; AD, Alzheimer's disease; ALA, $\alpha$-linolenic acid; ALOX5AP, arachidonate 5lipoxygenase activating protein; COX, cyclooxygenase; cPLA2, cytosolic phospholipase A2; CYP, cytochrome P450; DEGs, differentially expressed genes; DGLA, dihomo- $\gamma$-linolenic acid; DHA, docosahexaenoic acid; DiHETrE, dihydroxyeicosatrienoic acid; EET, epoxy-eicosatrienoic acids; EPA, eicosapentaenoic acid; EpDPE, epoxy-docosapentaenoic acid; EpOME, epoxy-octadecenoic acid; HEPE, hydroxyleicosapentaenoic acid; HETE, hydroxy-eicosatetraenoic acid; ICH, intracerebral hemorrhage; KEGG, Kyoto Encyclopedia of Genes and Genomes; LA, linoleic acid; LC/MS, liquid chromatography tandem mass spectrometry; LOX, lipoxygenase; OPLS-DA, orthogonal projections to latent structures; oxo-ETE, oxo-eicosatetraenoic acid; oxoODE, oxo-octadecadienoic acid; PCA, principal component analysis; PUFAs, polyunsaturated fatty acids; RNA-seq, RNA sequencing; sEH, soluble epoxide hydrolase; VIP, variable importance in projection. 


\section{INTRODUCTION}

Oxylipins are polyunsaturated fatty acid (PUFAs) oxidation products formed via one or more mono- or dioxygen-dependent reactions (Gabbs et al., 2015). These PUFAs consist of arachidonic acid (AA), docosahexaenoic acid (DHA), eicosapentaenoic acid (EPA), linoleic acid (LA), and $\alpha$-linolenic acid (ALA). Once liberated by cytosolic phospholipase A2 (cPLA2) from membrane phospholipids, free PUFAs can be metabolized to form oxylipins via auto-oxidation or enzymes including cyclooxygenase (COX), lipoxygenase (LOX), and cytochrome P450 (CYP)-soluble epoxide hydrolase (sEH) pathways (Caligiuri et al., 2017). Given their short half-time and low content, oxylipins are found all over the tissues and support the body's homeostasis by regulating many important physiological processes such as inflammation, blood coagulation, and vascular function (Nayeem, 2018).

Arachidonic acid and DHA are the major PUFAs in the brain, nevertheless the profile of oxylipins does not completely reflect the constitutions of PUFAs, but also depends on the oxygenases available and the oxygenases' affinity for a specific substrate PUFAs (Gabbs et al., 2015). Nearly half of the oxylipins in the brain are derived from AA, while less than $20 \%$ of oxylipins are produced from DHA which accounts for $40 \%$ of total PUFAs (Ferdouse et al., 2019). Thus comprehensive analysis of the oxylipin profile is necessary to clarify the constitutions and possible functions of oxylipins in the brain. There is also research focusing on some oxylipins or oxylipin related enzymes such as COX and 5/12/15-LOX which in acute experimental ICH model indicates that oxylipins are important regulatory factors of ICH (Wu et al., 2011; Rosso et al., 2014; Xu et al., 2017; Karuppagounder et al., 2018; Han et al., 2019). However, the specific profile of oxylipins and the overall expression pattern of their relative enzymes in $\mathrm{ICH}$ has not been fully discovered yet. On the other hand, different from ischemia stroke which shows masses of PUFAs released from brain parenchyma, erythrocytes entering the brain during ICH will be gradually lysed releasing substantial cell membrane for further metabolism. The constitution of PUFAs in blood is quite different with the brain, which may specifically affect the metabolism of certain oxylipins in ICH.

In order to comprehensively quantify the profile of oxylipins in $\mathrm{ICH}$, we constructed an ICH mouse model using autologous blood injection and the tissues around hematoma were collected in the acute phase of ICH. The concentrations of oxylipins were then detected via liquid chromatography tandem mass spectrometry (LC/MS). On the other hand, the mRNA levels of oxylipin relative enzymes were also reanalyzed based on our previously constructed RNA-sequence (RNA-seq) library of ICH. We found that the profile of oxylipins shifted as the progression of $\mathrm{ICH}$ and the metabolism of $\mathrm{AA}$ and EPA was highly affected in ICH. This study extends our understanding of the change in oxylipin profile and their enzymes in ICH, which will help explore the functions of oxylipins in ICH.

\section{MATERIALS AND METHODS}

\section{Animals}

As showed in the flow chart of the present study, a total of 40 mice were used for oxylipins detection (Figure 1). C57BL/6 mice (male, 8-week-old, 20-24g) were purchased from the Animal Center of the Army Medical University (Chongqing, China). The mice were raised in the specific pathogen-free grade environment with $12 \mathrm{~h}$ light $/ 2 \mathrm{~h}$ dark cycle and given ad libitum access to food and water. All procedures and animal experiments were approved and conducted in accordance with the Animal Ethics Committee of the Army Medical University.

\section{ICH Model}

The ICH model was conducted according to our previously developed method (Yuan et al., 2019). Briefly, mice were immobilized on the stereotaxic apparatus (RWD Life Science Co, Shenzhen, China) after being anesthetized with 3\% isoflurane for induction and $1.5 \%$ for maintenance. Whole blood $(20 \mu \mathrm{l})$ without anticoagulant was obtained from its tail and then injected into the left striatum $(0.8 \mathrm{~mm}$ anterior and $2 \mathrm{~mm}$ lateral of bregma, at a depth of $3.5 \mathrm{~mm}$ ) using a syringe pump (Hamilton, Bonaduz, AG) at $2.0 \mu \mathrm{l} / \mathrm{min}$. The microinjector was detained for $10 \mathrm{~min}$. The sham group was injected with $20 \mu$ saline using the same procedures. Unsuccessful ICH models that were asymptomatic or dead were excluded from this study. Perihematomal cerebral tissues were collected for oxylipins quantification at $0.5,1$, and 3 days after ICH or 1 day after saline injection.

\section{Oxylipin Extraction}

Oxylipin extraction was performed according to the previously developed method (Ostermann et al., 2015; Sun et al., 2019). Ultra-performance liquid chromatography (UPLC)-grade methanol, acetonitrile, acetic acid, isopropanol, hydrochloric acid ( $\mathrm{HCl})$, menthyl formate, n-hexane, and water were purchased from Merck (Darmstadt, GER) and the standard samples of all oxylipins were purchased from Cayman (Michigan, United States). Every $100 \mathrm{mg}$ of brain tissue was homogenized with $2 \mathrm{ml}$ of $4^{\circ} \mathrm{C}$ methanol solution. After $5 \mathrm{~min}$ of vortex, the sample was laced for protein precipitation at low temperature. Twenty microliter of $1 \mu \mathrm{M}$ internal standard mixture was added to each sample and centrifuged at $5000 \mathrm{rpm}$ for $10 \mathrm{~min}$. Supernatants were taken and dried within nitrogen. Eight milliliter of $10 \%$ methanol water was used as a dissolvent and the $\mathrm{pH}$ (3.5) was adjusted using $0.2 \mathrm{~mol} / \mathrm{l} \mathrm{HCl}$. The solid phase extraction column (SepPak tC18 SPE, $6 \mathrm{ml}, 500 \mathrm{mg}, 37-55 \mu \mathrm{m}$ particle, waters) was equilibrated by $5 \mathrm{ml}$ methanol followed by $5 \mathrm{ml}$ water. Samples with appropriate $\mathrm{pH}$ were then transferred to the prepared solid phase extraction column. The column was rinsed with $4 \mathrm{ml}$ water followed by $5 \mathrm{ml} \mathrm{n}$-hexane. Elution was performed using $10 \mathrm{ml}$ menthyl formate and the eluates were collected. Eluates were blown dry with nitrogen, then reconstituted with $200 \mu \mathrm{l}$ methanol-water $(\mathrm{v}: \mathrm{v}=1: 1)$ for $30 \mathrm{~s}$, and the supernatants were then available for oxylipins analysis. 


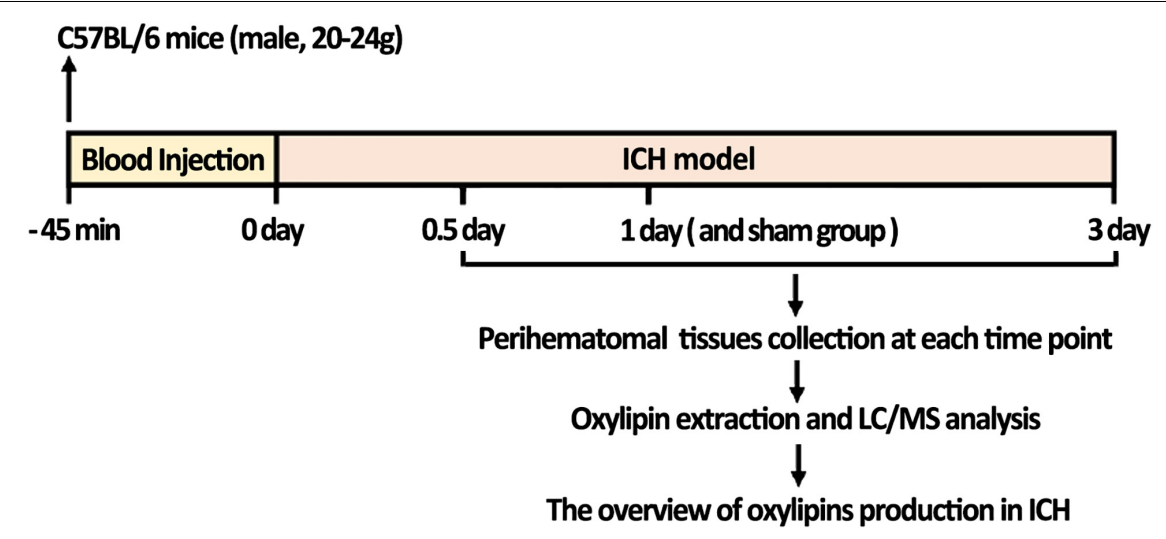

FIGURE 1 | The flow chart of the study; ICH mice were constructed and collected 0.5, 1, and 3 days after ICH. LC-MS/MS analysis was then performed to quantify the change of oxylipins in the brain. Mice injected with saline and collected after 1 day were used as the sham control.

\section{Liquid Chromatography and Mass Spectrometry}

Liquid chromatography tandem mass spectrometry was performed according to the previously developed method (Willenberg et al., 2015). Briefly, the sample extracts were analyzed using an LC-ESI-MS/MS system (UPLC, Shim-pack UFLC SHIMADZU CBM A system, https://www.shimadzu.com/; MS, QTRAP ${ }^{\circledR}$ 6500+ System, https://sciex.com/). The analytical conditions were as follows, UPLC: column, ACQUITY UPLC HSS T3 $(1.8 \mu \mathrm{m}$, $2.1 \mathrm{~mm} \times 100 \mathrm{~mm}$ ); solvent system, A: acetonitrile/water $(60 / 40 \mathrm{~V}, 0.02 \%$ acetic acid), B: acetonitrile / isopropanol (50/50 $\mathrm{V})$; gradient program, $\mathrm{A} / \mathrm{B}(99.9: 0.1 \mathrm{~V} / \mathrm{V})$ at $0 \mathrm{~min}, 70: 30 \mathrm{~V} / \mathrm{V}$ at $2.0 \mathrm{~min}, 50: 50 \mathrm{~V} / \mathrm{V}$ at $4 \mathrm{~min}, 1: 99 \mathrm{~V} / \mathrm{V}$ at $5.5 \mathrm{~min}, 1: 99 \mathrm{~V} / \mathrm{V}$ at $6.0 \mathrm{~min} ; 99.9: 0.1 \mathrm{~V} / \mathrm{V}$ at $7.0 \mathrm{~min}$; stop at $10.0 \mathrm{~min}$; flow rate: $0.40 \mathrm{ml} / \mathrm{min}$; temperature: $40^{\circ} \mathrm{C}$; injection volume: $10 \mu \mathrm{l}$. The effluent was alternatively connected to an ESI-triple quadrupolelinear ion trap (QTRAP)-MS. LIT and triple quadrupole (QQQ) scans were acquired on a triple quadrupole-linear ion trap mass spectrometer (QTRAP), QTRAP ${ }^{\circledR}$ 6500+ LC-MS/MS System, equipped with an ESI Turbo Ion-Spray interface, operating in negative ion mode and controlled by Analyst 1.6.3 software (Sciex). The ESI source operation parameters were as follows: ion source, turbo spray; source temperature $550^{\circ} \mathrm{C}$; Multiple reaction monitoring (MRM) scan, negative ion mode; EPI scan range: $m / z: 50-640$; scan speed: $1000 \mathrm{da} / \mathrm{s}$; curtain gas, ion source gas 1 and ion source gas 2 were delivered at 35,40 , and $40 \mathrm{psi}$, respectively. These jobs were completed with the help of the Wuhan Metware Biotechnology Co. Ltd. (Wuhan, China).

\section{Statistical Analysis}

Unsupervised principal component analysis (PCA) was performed by R. The cluster analysis results of metabolites were presented as heatmaps. Differential analysis was performed to compare the content of the $0.5,1$, and 3 days ICH groups to the sham group using Orthogonal projections to latent structures (OPLS-DA) by metaboanalyst software and the MannWhitney test. Variable importance in projection (VIP) values were extracted from OPLS-DA results, which also contained score plots and permutation plots, generated using the $\mathrm{R}$ package. Significantly produced metabolites between groups were determined by VIP $\geq 1, p<0.05$ using the Mann-Whitney $\mathrm{U}$ test and absolute $\log _{2} \mathrm{FC} \geq 1$ or $\leq 0.5$ (Sun et al., 2019). The identified metabolites were annotated using the Kyoto Encyclopedia of Genes and Genomes (KEGG) database, and the annotated metabolites were then mapped to the KEGG pathway database. Pathways with significantly produced metabolites were then fed into metabolite sets enrichment analysis, the significance of which was determined by the $p$-value.

\section{RNA-Seq Dataset Processing}

The RNA-seq dataset was previously constructed and based on 13-month and 22-month-old SD rats using the contralateral hemisphere as control at 3 days after ICH. To analyze the expression of oxylipins relative enzymes in ICH, all of the cPLA2, COX, LOX, CYP, and sEH were first selected. Their raw DNA reads were first discarded if there were more than $2-\mathrm{N}$ base reads, clipping adaptor, low quality bases (less than 20), and too short (less than $16 \mathrm{nt}$ ) using the Cutadapt software according to a previous study (Kechin et al., 2017). After that, the reads were compared to the Rattus norvegicus genome sequences (Rnor 6.0) and annotation file (Rnor 6.0.82) from the Ensembl database ${ }^{1}$. After, the reads were aligned to the rat genome using TopHat2 according to a previous study with an end-to-end method allowing two mismatches (Kim et al., 2013). The aligned DNA reads with more than one genomic localization were also discarded and the uniquely localized reads were calculated for the read number and RPKM value (The reads per kilobase and per million). We then obtained the gene coverage and depth, read distribution around transcription starting sites, and transcription terminal sites. After attaining the level of gene expression in all samples, we also obtained the differentially expressed genes (DEGs) using edgeR, one of the R packages, according to a previous study which compared the adjacent time point samples to investigate the genes that were regulated by

\footnotetext{
${ }^{1}$ http://asia.ensembl.org/Rattus_norvegicus/Info/Index
} 
aging or by comparing the treated and normal ICH samples at the same time points (Robinson et al., 2010). For each gene, we also obtained the p-value according to the model of the negative binomial distribution and estimated the fold change using this package when $p$-value $<0.01$ and 2 -fold changes $\left(\log _{2} \mathrm{FC}>1\right)$ were reached.

\section{RESULTS}

\section{Oxylipin Profile in the Acute Phase of Intracerebral Hemorrhage}

To gain better insight into the full spectrum of oxylipins alterations initiated by ICH at the molecular level, LC/MS was performed on the brain samples obtained from mice sacrificed at $0.5,1$, and 3 days following ICH and 1 day after saline injection for the sham group (Figure 1). In this study, 58 oxylipins were quantifiable in the brain (Figure 2A and Supplementary Table S1). Consistent with other studies on the profile of cerebral oxylipins, nearly half of oxylipins were derived from AA (48.3\%). EPA derived oxylipins ranked second (13.8\%), followed by DHA (12.1\%), LA (10.3\%), DGLA (8.6\%), $\alpha$-LA (3.5\%), and $\gamma$-LA (3.5\%) (Figure 2A). Based on the assessment of PCA, the profile of oxylipins in $\mathrm{ICH}$ was shifted compared to the sham group, and the differences in 1 day seemed to be the biggest, while the profile at 3 days was largely overlapped with the sham group (Figure 2B). Further OPLS-DA analysis showed that the levels of 17 oxylipins were increased and none were significantly decreased in $\mathrm{ICH}$ (Table 1). Most of the increased oxylipins in $\mathrm{ICH}$ were derived from AA (10/17) and EPA (4/17), followed by LA (13-oxoODE; 9,10-EpOME) and DHA (16,17-EpDPE) (Table 1). With respect to oxylipins' patterns, 4 out of 8 oxylipins from EPA and 10 out of 28 oxylipins from AA were found increased in the acute phase of ICH indicating that the metabolism of AA and EPA was highly affected in ICH (Figure 2A).

Among the increased oxylipins in ICH, LTE4, 5,6-DiHETrE, PGJ2, 5-oxoETE, and 8,9-EET were the top five metabolites that increased most from AA in ICH (Figure 3A); 12-HETE, an important effector of AA, also showed an increased trend in $\mathrm{ICH}$ and was significant increased 1 day following ICH (Figure 3A); 18-HEPE belonging to EPA was increased throughout the whole acute phase of ICH (Figure 3B); 16,17-EpDPE, the only increased DHA derived oxylipin returned to normal 3 days after $\mathrm{ICH}$ (Supplementary Figure S1A).

Furthermore, the profile of oxylipins shifted with the progression of ICH. Eight of 17 increased oxylipins had already increased 0.5 day after the injection of blood and kept increasing to 14 after 1 day, but these oxylipins went back to normal rapidly with only 5 metabolites that were increased 3 days after $\mathrm{ICH}$, which matched the trends seen in PCA (Table 1). Defined by the fold change, 12-HEPE, 16,17-EpDPE, 8,9-EET, 5,6-DiHETrE, and 18-HEPE were the top five metabolites that increased most in the ICH 0.5 day group and they kept increasing 1 day after $\mathrm{ICH}$ with LTE4 ranked first (Table 1). However, PGJ2, 15-oxo-ETE, 12-HEPE, 18-HEPE, and 5-oxo-ETE were the only oxylipins that increased 3 day after ICH suggesting that the profile of oxylipin at 3 days after ICH was different with the former (Table $\mathbf{1}$ ).
Further KEGG analysis provided clues regarding the causes of changes in the oxylipin profile. The KEGG pathway of AA metabolism at 0.5 and 1 days after $\mathrm{ICH}$ revealed that most of the increased oxylipins were produced by CYP and LOX (Figure 3C and Supplementary Figure S1B). Oxylipins produced by COX were more predominant 3 days after $\mathrm{ICH}$, suggesting that the activation of COX might be slower than the CYP and LOX pathways (Supplementary Figure S1C). Other oxylipins also showed similar characteristics. Unlike LOX that was activated through the whole experiment, most products of CYP including 16,17-EpDPE from DHA and 9,10-EpOME from LA returned to normal at 3 days after ICH. These results might hint that the profile of oxylipins in $\mathrm{ICH}$ might relate to the activation and selectivity of their enzymes.

\section{The Expression of Oxylipin Relative Enzymes in ICH}

The RNA-seq dataset was previously constructed and was based on 13-month and 22-month-old SD rats at 3 days after ICH, which corresponded with the common age of ICH. A total of 90 oxylipin relative molecules were quantifiable in the brain except for COX (Figure 4A). The expression patterns of oxylipins relative enzymes were extremely similar in both the 13-month and 22-month-old ICH group and CYP was the most affected by ICH (Figure 4A). Among these, Cyp4f18, Cyp1b1, and Cyp2d3 was found up-regulated in both the 13-month and 22-month groups after $\mathrm{ICH}$, except that $\mathrm{Cyp} 2 e 1$ was mainly increased in the 22-month group and Cypla1 was mainly increased in the younger group (Figure 4B). Cyp26b1 was found to have a decreased trend in $\mathrm{ICH}$ and was more pronounced in the younger group (Figure 4B). In the cluster of LOX, ALOX5AP was showed to have two times up-regulated and PLA2g4a was the only cPLA2 that kept increased in ICH (Figure 4B). In addition, there was no significant change of $s E H$ in ICH (Figure 4A).

\section{DISCUSSION}

This study provides foundational data concerning on the alterations of oxylipins profile in ICH by quantitative LC/MS. Fifty-eight oxylipins were quantifiable in the study and nearly half of oxylipins were derived from AA in either the sham or ICH group, which was similar with the feature of cerebral oxylipins (Ferdouse et al., 2019). Different to other research, EPA derived oxylipins comprised $13.8 \%$ of the profile of cerebral oxylipins, which was a slightly larger proportion than that accounted for by DHA-derived oxylipins. The disparity might be caused by the differences in databases where some DHA derived oxylipins were missed during LC/MS analysis. Hitherto, there is still no standard oxylipins database being published and the biggest database about cerebral oxylipins was constructed by Harold $M$. Aukema, which gauged 87 oxylipins in the brain (Ferdouse et al., 2019). Given that most of the content of oxylipins are extremely low in the tissues, methods with higher accuracy and sensitivity are urgently needed.

OPLS-DA analysis showed that 17 out of 58 oxylipins were significantly increased and none were decreased in ICH. Among 

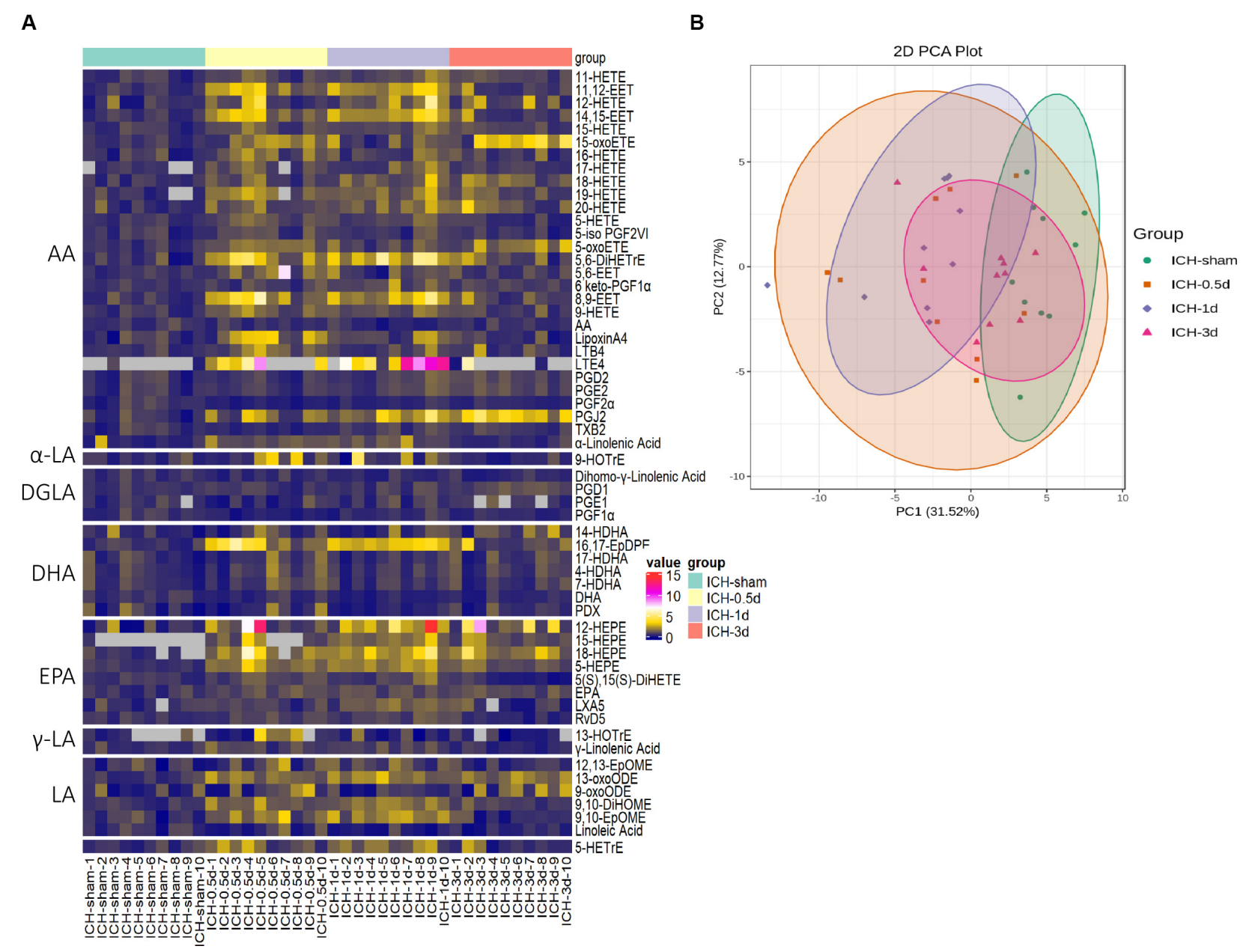

FIGURE 2| The profile of oxylipins in the acute phase of ICH. (A) The hierarchical cluster of the heat map shows the profile of oxylipins in ICH $(n=10)$. (B) Principal component analysis (PCA) of the oxylipins in four groups $(n=10)$.

the quantifiable oxylipins from EPA and AA in the brain, 4/8 and $10 / 28$ were increased in $\mathrm{ICH}$, respectively, which was higher than other clusters of oxylipins. In this respect, the metabolism of EPA and AA was mostly affected in ICH. However, it is interesting to note that the brain maintains very low levels of EPA but contains high levels of DHA (Chen et al., 2013). Besides, masses of oxylipins are quickly produced and peaked after the first day of ICH. These up-regulated oxylipins return to normal rapidly except for 12-HEPE and 18-HEPE, while the five oxylipins that increased 3 days following ICH indicate that the profile of oxylipins shifted with the progression of ICH. Interestingly, the regular pattern of oxylipins corresponded with the lysis of RBCs in hematoma. The lysis of RBCs relies on the phagocytosis of microglia/macrophages in parenchyma and compared to 1 day after ICH, most of the RBCs in hematoma are absorbed as the activation and infiltration of microglia/macrophages occurs 3-7 days following ICH (Fang et al., 2014; Zhao et al., 2015; Wang et al., 2017, 2018) 2018). Therefore, the lysis of RBCs may cause more dramatic effects on the production of oxylipins with the progression of ICH until the hematoma is completely absorbed. For these reasons, we infer that the phospholipids from RBCs of hematoma are another source for the production of oxylipins in the acute phase of $\mathrm{ICH}$, especially those derived from EPA. To further prove this hypothesis, it is nececessary to compare the increase in brain lipids to the fatty acid and oxylipin levels in blood, and metabolic flux isotope analysis is warranted in the future.

The functions of oxylipins involve many aspects of body homeostasis including innate immunity, inflammation, cardiac function, blood coagulation, and vascular tone regulation (Nayeem, 2018). Compared to oxylipins derived from n-3 PUFAs (DHA, EPA), those derived from n-6 PUFAs (AA, LA) generally, but not always, exhibit pro-inflammatory, vasoconstrictive, and proliferative effects (Gabbs et al., 2015). In the acute phase of ICH, the increased production of LTE4, PGJ2, 5-oxo-ETE, and 15-oxo-ETE derived from AA may exhibit vasoconstrictive, pro-apoptotic, pro-inflammatory, and chemoattractant effects which are inclined to aggregate the damage of $\mathrm{ICH}$, while 5,6-DiHETrE, 12-HETE, and EETs show opposing effects such as vasodilative, anti-apoptotic, or anti-inflammatory activities 
TABLE 1 | Up-regulated oxylipins in the acute phase of $\mathrm{ICH}$.

\begin{tabular}{|c|c|c|c|c|c|c|c|c|c|c|c|c|c|}
\hline \multirow[t]{2}{*}{ Oxylipin } & \multirow{2}{*}{$\frac{\text { Sham }}{\text { Content }(\mathrm{nmol} / \mathrm{g})}$} & \multicolumn{4}{|c|}{ ICH 0.5 day } & \multicolumn{4}{|c|}{ ICH 1 day } & \multicolumn{4}{|c|}{ ICH 3 days } \\
\hline & & Content (nmol/g) & VIP & $p$-value & $\log _{2} F C$ & Content (nmol/g) & VIP & $p$-value & $\log _{2} F C$ & Content $(\mathrm{nmol} / \mathrm{g})$ & VIP & $p$-value & $\log _{2} F C$ \\
\hline \multicolumn{14}{|l|}{ AA } \\
\hline LTE4 & $0.022 \pm 0.011$ & $0.104 \pm 0.049$ & 0.97 & N/A & 2.24 & $0.155 \pm 0.089^{\star}$ & 1.41 & N/A & 2.81 & $0.053 \pm 0.069$ & 0.32 & $\mathrm{~N} / \mathrm{A}$ & 1.27 \\
\hline 5,6-DiHETrE & $0.004 \pm 0.002$ & $0.010 \pm 0.005^{\star}$ & 1.44 & 0.00 & 1.48 & $0.013 \pm 0.005^{\star}$ & 1.46 & 0.00 & 1.84 & $0.006 \pm 0.003$ & 0.99 & 0.03 & 0.71 \\
\hline 8,9-EET & $0.021 \pm 0.007$ & $0.062 \pm 0.032^{*}$ & 1.51 & 0.00 & 1.56 & $0.062 \pm 0.023^{\star}$ & 1.55 & 0.00 & 1.56 & $0.030 \pm 0.013$ & 1.11 & 0.08 & 0.51 \\
\hline 14,15-EET & $0.071 \pm 0.018$ & $0.171 \pm 0.063^{*}$ & 1.50 & 0.00 & 1.26 & $0.204 \pm 0.051^{*}$ & 1.60 & 0.00 & 1.51 & $0.094 \pm 0.048$ & 0.70 & 0.19 & 0.39 \\
\hline 11,12-EET & $0.054 \pm 0.013$ & $0.133 \pm 0.048^{*}$ & 1.53 & 0.00 & 1.30 & $0.148 \pm 0.035^{\star}$ & 1.59 & 0.00 & 1.45 & $0.070 \pm 0.033$ & 0.75 & 0.19 & 0.37 \\
\hline PGJ2 & $1.159 \pm 0.458$ & $2.257 \pm 1.039$ & 1.01 & 0.01 & 0.96 & $3.033 \pm 1.343^{\star}$ & 1.31 & 0.00 & 1.39 & $3.845 \pm 1.086^{\star}$ & 1.91 & 0.00 & 1.73 \\
\hline 12-HETE & $3.685 \pm 2.032$ & $6.344 \pm 4.513$ & 0.99 & 0.11 & 0.78 & $8.100 \pm 5.020^{\star}$ & 1.22 & 0.02 & 1.14 & $6.100 \pm 3.300$ & 1.12 & 0.07 & 0.73 \\
\hline LipoxinA4 & $0.003 \pm 0.001$ & $0.006 \pm 0.003^{*}$ & 1.11 & 0.00 & 1.25 & $0.004 \pm 0.002$ & 0.74 & 0.04 & 0.66 & $0.003 \pm 0.001$ & 0.04 & 0.85 & 0.05 \\
\hline 15-OXOETE & $0.048 \pm 0.011$ & $0.096 \pm 0.033$ & 1.30 & 0.00 & 0.99 & $0.071 \pm 0.035$ & 0.62 & 0.07 & 0.56 & $0.148 \pm 0.061^{*}$ & 1.73 & 0.00 & 1.61 \\
\hline 5-OXOETE & $0.101 \pm 0.024$ & $0.184 \pm 0.033$ & 1.53 & 0.00 & 0.87 & $0.140 \pm 0.044$ & 0.86 & 0.03 & 0.48 & $0.209 \pm 0.078^{\star}$ & 1.68 & 0.00 & 1.06 \\
\hline \multicolumn{14}{|l|}{ EPA } \\
\hline 12-HEPE & $0.079 \pm 0.061$ & $0.256 \pm 0.303$ & 1.20 & 0.10 & 1.69 & $0.308 \pm 0.290^{*}$ & 1.37 & 0.04 & 1.96 & $0.236 \pm 0.193^{\star}$ & 1.39 & 0.03 & 1.58 \\
\hline 18-HEPE & $0.004 \pm 0.001$ & $0.012 \pm 0.006^{*}$ & 1.00 & 0.01 & 1.46 & $0.012 \pm 0.005^{\star}$ & 1.24 & 0.00 & 1.52 & $0.010 \pm 0.005^{\star}$ & 1.62 & 0.00 & 1.30 \\
\hline 5-HEPE & $0.005 \pm 0.001$ & $0.011 \pm 0.003^{*}$ & 1.67 & 0.00 & 1.09 & $0.012 \pm 0.002^{*}$ & 1.64 & 0.00 & 1.18 & $0.008 \pm 0.002$ & 1.69 & 0.00 & 0.63 \\
\hline 15-HEPE & $0.003^{a}$ & $0.005 \pm 0.002$ & 1.20 & $\mathrm{~N} / \mathrm{A}$ & 0.88 & $0.005 \pm 0.001^{\star}$ & 1.57 & $\mathrm{~N} / \mathrm{A}$ & 1.01 & $0.004 \pm 0.002$ & 2.04 & $\mathrm{~N} / \mathrm{A}$ & 0.70 \\
\hline \multicolumn{14}{|l|}{ DHA } \\
\hline 16,17-EpDPE & $0.030 \pm 0.008$ & $0.092 \pm 0.046^{\star}$ & 1.48 & 0.00 & 1.62 & $0.098 \pm 0.012^{\star}$ & 1.63 & 0.00 & 1.71 & $0.048 \pm 0.028$ & 1.06 & 0.07 & 0.68 \\
\hline \multicolumn{14}{|l|}{ LA } \\
\hline 13-oxoODE & $0.010 \pm 0.003$ & $0.018 \pm 0.004$ & 1.48 & 0.00 & 0.96 & $0.020 \pm 0.005^{\star}$ & 1.40 & 0.00 & 1.11 & $0.019 \pm 0.004$ & 1.72 & 0.00 & 0.98 \\
\hline 9,10-EpOME & $0.004 \pm 0.002$ & $0.009 \pm 0.003$ & 1.16 & 0.00 & 0.98 & $0.010 \pm 0.003^{\star}$ & 1.23 & 0.00 & 1.21 & $0.004 \pm 0.002$ & 0.01 & 0.84 & -0.06 \\
\hline
\end{tabular}

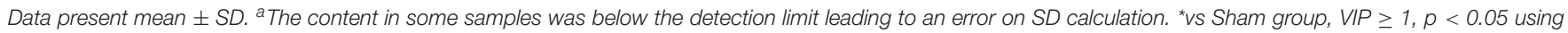
the Mann-Whitney $U$ test and $\log _{2} F C \geq 1$. VIP values were calculated by an OPLS-DA model using the metaboanalyst software.

(Sudhahar et al., 2010; Bennett and Gilroy, 2016; FigueiredoPereira et al., 2016; Shearer and Walker, 2018; Rahman et al., 2019; Wójcik et al., 2020). Of note, these oxylipins of AA change with the progression of ICH and the destructive oxylipins appear to peak 3 days after ICH when the protective oxylipins of AA have descended suggesting that AA-derived oxylipins tend to cause damage with the development of ICH. Studies show that HEPE tends to present anti-inflammatory effects and 12-HEPE can improve glucose metabolism by promoting glucose uptake through the insulin-like intracellular signaling pathway (Leiria et al., 2019). 18-HEPE, the precursor of the E-series specialized pro-resolving mediators, is another inhibitor of inflammation, and can halt the development of maladaptive cardiac remodeling and atherosclerosis (Endo et al., 2014; Liu et al., 2018). 16,17EpDPE derived from DHA is a vasodilator which regulates the calcium-potassium (BK) channel in arterial smooth muscle cells and correlates with the decrease of white matter hyperintensity in older populations with a high risk of dementia (Wang et al., 2011; Shinto et al., 2020). These properties of EPA- and DHAderived oxylipins suggest that they are potential defenders in $\mathrm{ICH}$. However, direct evidence is urgently needed to explore the functions of up-regulated oxylipins in $\mathrm{ICH}$.

There is still no research providing full information on the expression pattern of oxylipin relative enzymes in $\mathrm{ICH}$, thus transcriptome studies related to ICH such as RNA-seq and microarray can be an alternative source. Nevertheless, only a few studies contain such information and the profiles of oxylipins relative enzymes in each study are different
(Carmichael et al., 2008; Rosell et al., 2011; Sang et al., 2017; Walsh et al., 2019). Some researchers have also used peripheral blood mononuclear cells as a source of mRNA for gene expression profile analysis instead of perihematomal tissues, but these studies do provide important clues on the change in oxylipin relative enzymes in ICH (Sang et al., 2017; Walsh et al., 2019). Substantial peripheral inflammatory cells will infiltrate into the brain through the breakdown of the blood-brain barrier (BBB) and are involved in the $\mathrm{ICH}$-induced inflammatory response (Xiong and Yang, 2015). These inflammatory cells that are full of oxylipin relative enzymes are involved in the lysis of RBCs and thus may be closely linked to the change of oxylipins in $\mathrm{ICH}$. Consistent with our results, ALOX5AP is found to increase in the acute phase of ICH (Rosell et al., 2011; Walsh et al., 2019). Besides, there are two studies, whose samples are taken during $3 \mathrm{~h}$ and $24 \mathrm{~h}$ after the onset of ICH, showing that ALOX5AP and Cyp1b1 is significant increased in this very early stage of $\mathrm{ICH}$ (Carmichael et al., 2008; Sang et al., 2017). This may hint that the up-regulation of ALOX5AP and Cyp1b1 can sustain through the acute phase of ICH.

ALOX5AP is associated with the development of $\mathrm{ICH}$, but the effect of ALOX5AP on ICH is as yet undiscovered (Kim et al., 2011). ALOX5AP is closely linked with ALOX5 and they are both required for leukotriene synthesis. Their metabolites include LTE4 and 5-oxo-ETE which exhibits proapoptotic or pro-inflammatory effects and targeting ALOX5 can effectively reduce ferroptosis and improve outcomes following $\mathrm{ICH}$, which makes us wondering if ALOX5AP could regulate 


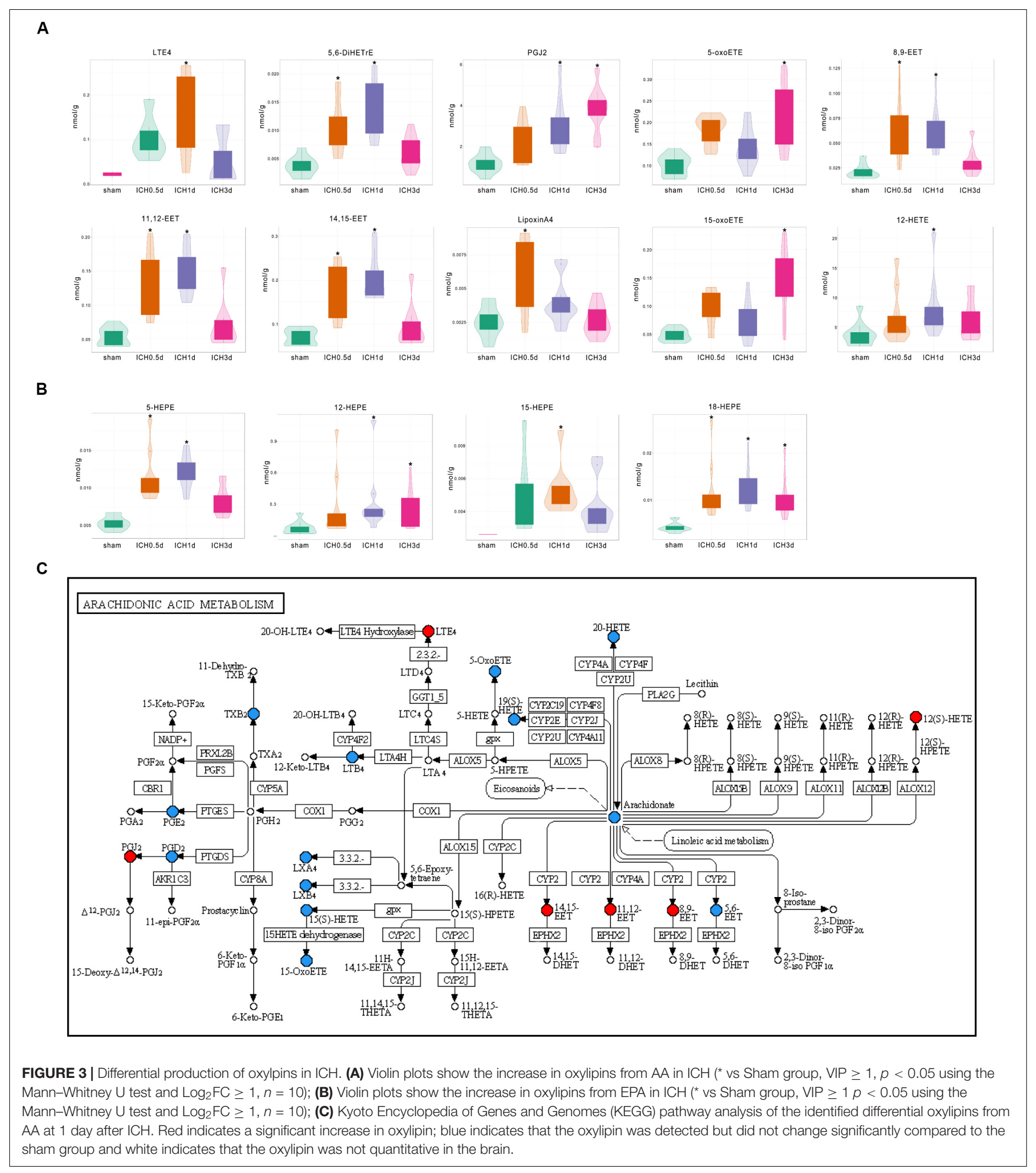

the damage of ICH (Postula et al., 2016; Karuppagounder et al., 2018). Other detected enzymes may also be linked to the over production of oxylipins in ICH. For example, Cyp2 is inclined to participate in DHA metabolism and may be responsible for the production of 16,17-EpDPE (Wang et al., 2011). Cyp4 is the putative metabolic enzyme of 18-HEPE and ALOX12, upregulated in the early stage of $\mathrm{ICH}$, is responsible for $12-\mathrm{HEPE}$ production (Kalsotra et al., 2004). However, there remain many oxylipins, such as 5,6-DiHETrE that are not directily linked with given enzymes, so future studies are needed to verify 
A

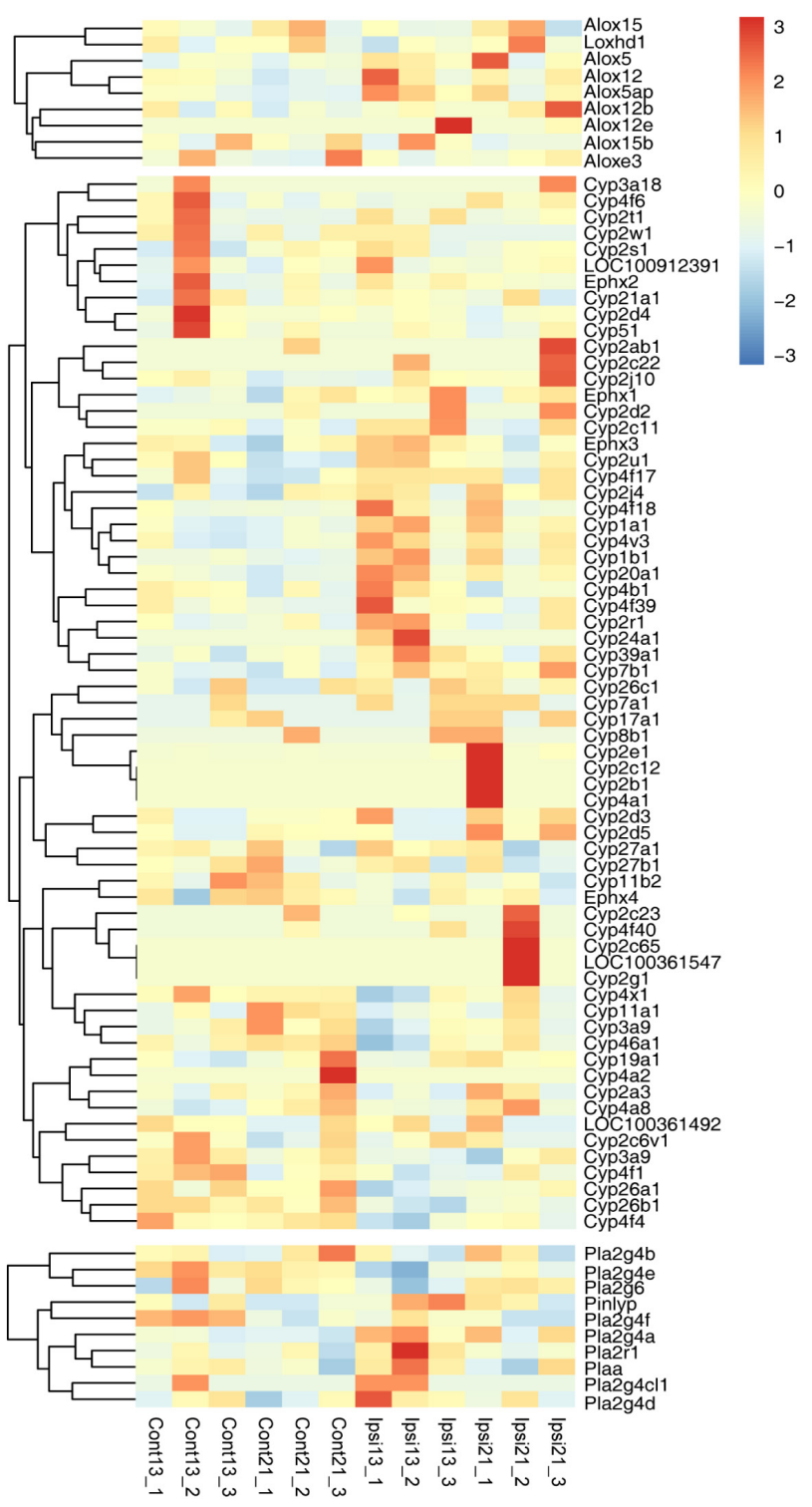

B
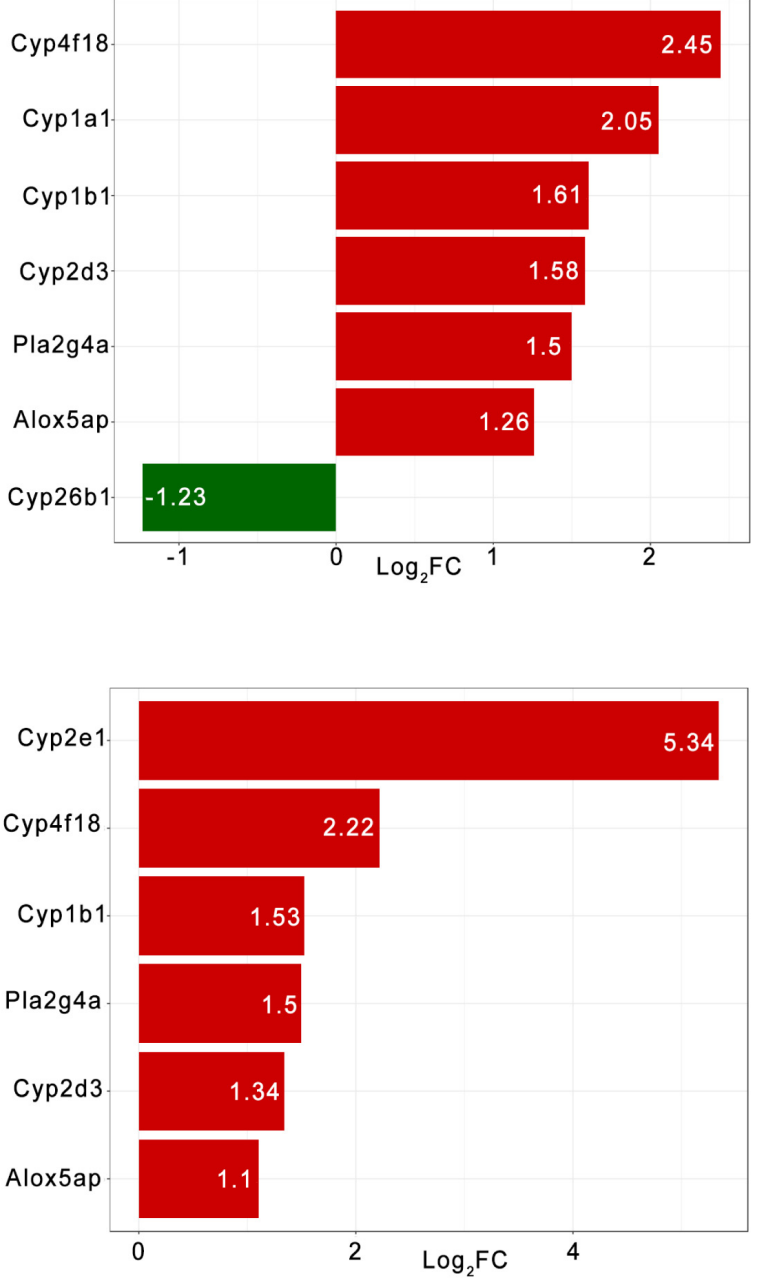

FIGURE 4 | The expression pattern of oxylipins relative enzymes in $\mathrm{ICH}$. (A) The hierarchical cluster of the heat map shows the expression of oxylipins related enzymes in ICH $(n=3)$; (B) Differential expression of oxylipins related enzymes in 13-month-old ICH rats (top panel) and 22-month-old ICH rats (bottom panel) (vs contralateral hemisphere, $p<0.01$ and $\log _{2} \mathrm{FC}>1, n=3$ ).

the relationship between metabolite enzymes and oxylipins. An important limitation of the RNA-seq data is the mismatch between the model used for oxylipins and the RNA-seq. The 13month and 22-month-old SD rats were selected because age is an independent risk factor for ICH and most ICH happens in older populations. The 13-month and 22-month-old rats may better reflect the real situation of $\mathrm{ICH}$ but create certain biases in understanding of the relationship between oxylipins and their relative enzymes.

Despite these limitations, this is the first study to have comprehensively assessed the changes of oxylipins and their relative enzymes in $\mathrm{ICH}$. The profile of oxylipins shifted with the progression of $\mathrm{ICH}$ and the metabolism of AA and EPA was highly affected in ICH. A few specific oxylipins have been discovered in $\mathrm{ICH}$, which are worth exploring in future studies to identify the functions and regulatory mechanisms and to determine whether these oxylipins can be potential targets for the treatment of ICH.

\section{DATA AVAILABILITY STATEMENT}

The RNA-seq dataset was uploaded at Gene Expression Omnibus (GEO) with accession number GSE149317. 


\section{ETHICS STATEMENT}

The animal study was reviewed and approved by Animal Ethics Committee of the Army Medical University.

\section{AUTHOR CONTRIBUTIONS}

J-JY and Q-WY conceptualized this study and designed the experiments. J-JY, QC, QZ, and J-CH established the $\mathrm{ICH}$ models. J-JY, G-QY, C-XG, and Z-MQ performed the oxylipins quantification and data analysis. W-JZ, H-FS, QH, and RX analyzed and interpreted the results of the RNA-seq experiments. QX and X-YX drafted the manuscript. Q-WY supervised the study. All authors approved the final version of the manuscript.

\section{FUNDING}

This study was supported by a grant from the National Natural Science Foundation of China (81901236).

\section{REFERENCES}

Bennett, M., and Gilroy, D. W. (2016). Lipid Mediators in Inflammation. Microbiol. Spectr. 4:a016311. doi: 10.1128/microbiolspec.MCHD-0035-2016

Caligiuri, S. P. B., Parikh, M., Stamenkovic, A., Pierce, G. N., and Aukema, H. M. (2017). Dietary Modulation of Oxylipins in Cardiovascular Disease and Aging. Am. J. Physiol. Heart Circ. Physiol. 313, H903-H918. doi: 10.1152/ajpheart. 00201.2017

Carmichael, S. T., Vespa, P. M., Saver, J. L., Coppola, G., Geschwind, D. H., Starkman, S., et al. (2008). Genomic profiles of damage and protection in human intracerebral hemorrhage. J. Cereb. Blood Flow Metab. 28, 1860-1875. doi: $10.1038 /$ jcbfm.2008.77

Chen, C. T., Domenichiello, A. F., Trépanier, M. O., Liu, Z., Masoodi, M., and Bazinet, R. P. (2013). The low levels of eicosapentaenoic acid in rat brain phospholipids are maintained via multiple redundant mechanisms. J. Lipid Res. 54, 2410-2422. doi: 10.1194/jlr.M038505

Endo, J., Sano, M., Isobe, Y., Fukuda, K., Kang, J. X., Arai, H., et al. (2014). 18-HEPE, an n-3 fatty acid metabolite released by macrophages, prevents pressure overload-induced maladaptive cardiac remodeling. J. Exp. Med. 211, 1673-1687. doi: 10.1084/jem.20132011

Fang, H., Chen, J., Lin, S., Wang, P., Wang, Y., Xiong, X., et al. (2014). CD36mediated hematoma absorption following intracerebral hemorrhage: negative regulation by TLR4 signaling. J. Immunol. 192, 5984-5992. doi: 10.4049/ jimmunol.1400054

Ferdouse, A., Leng, S., Winter, T., and Aukema, H. M. (2019). The Brain Oxylipin Profile Is Resistant to Modulation by Dietary n-6 and n-3 Polyunsaturated Fatty Acids in Male and Female Rats. Lipids 54, 67-80. doi: 10.1002/lipd.12122

Figueiredo-Pereira, M. E., Corwin, C., and Babich, J. (2016). Prostaglandin J2: a potential target for halting inflammation-induced neurodegeneration. Ann. N. Y. Acad. Sci. 1363, 125-137. doi: 10.1111/nyas.12987

Gabbs, M., Leng, S., Devassy, J. G., Monirujjaman, M., and Aukema, H. M. (2015). Advances in Our Understanding of Oxylipins Derived from Dietary PUFAs. Adv. Nutr. 6, 513-540. doi: 10.3945/an.114.00 7732

Han, X., Zhao, X., Lan, X., Li, Q., Gao, Y., Liu, X., et al. (2019). 20-HETE synthesis inhibition promotes cerebral protection after intracerebral hemorrhage without inhibiting angiogenesis. J. Cereb. Blood Flow Metab. 39, 1531-1543. doi: 10. $1177 / 0271678 X 18762645$

Kalsotra, A., Turman, C. M., Kikuta, Y., and Strobel, H. W. (2004). Expression and characterization of human cytochrome P450 4F11: Putative role in the

\section{ACKNOWLEDGMENTS}

We would like to thank the Wuhan Metware Biotechnology Co., Ltd. (Wuhan, China) for oxylipins quantification and ABLife Inc. (Wuhan, China) for the analysis of the RNA-seq dataset in this study.

\section{SUPPLEMENTARY MATERIAL}

The Supplementary Material for this article can be found online at: https://www.frontiersin.org/articles/10.3389/fnins. 2020.00777/full\#supplementary-material

FIGURE S1 | Differential production of oxylipin in ICH. (A) Violin plots show the increase in oxylipins from DHA (16,17-EpDPE) and LA (13-oxoODE, 9,10-EpOME) in $\mathrm{ICH}$ (* vs Sham group, VIP $\geq 1, p<0.05$ using the Mann-Whitney $U$ test and $\left.\log _{2} F C \geq 1, n=10\right) ;(\mathbf{B}, \mathbf{C})$ KEGG analysis of the identified differential oxylipins from $A A$ at 0.5 day $(\mathbf{B})$ and 3 days $(\mathbf{C})$ after $\mathrm{ICH}$. Red indicates a significant increase in oxylipin; blue indicates that the oxylipin was detected but did not change significantly and white indicates that the oxylipin was not quantitative in the brain.

metabolism of therapeutic drugs and eicosanoids. Toxicol. Appl. Pharmacol. 199, 295-304. doi: 10.1016/j.taap.2003.12.033

Karuppagounder, S. S., Alin, L., Chen, Y., Brand, D., Bourassa, M. W., Dietrich, K., et al. (2018). N-acetylcysteine targets 5 lipoxygenase-derived, toxic lipids and can synergize with prostaglandin $\mathrm{E}(2)$ to inhibit ferroptosis and improve outcomes following hemorrhagic stroke in mice. Ann. Neurol. 84, 854-872. doi: 10.1002/ana.25356

Kechin, A., Boyarskikh, U., Kel, A., and Filipenko, M. (2017). cutPrimers: A New Tool for Accurate Cutting of Primers from Reads of Targeted Next Generation Sequencing. J. Comput. Biol. 24, 1138-1143. doi: 10.1089/cmb.2017.0096

Kim, D., Pertea, G., Trapnell, C., Pimentel, H., Kelley, R., and Salzberg, S. L. (2013). TopHat2: accurate alignment of transcriptomes in the presence of insertions, deletions and gene fusions. Genome Biol. 14:R36. doi: 10.1186/gb-2013-14-4r36

Kim, D. H., Ahn, W. Y., Kim, D. K., Choe, B. K., Kim, S. K., Jo, D. J., et al. (2011). A Promoter polymorphism (rs17222919, -1316T/G) of ALOX5AP is associated with intracerebral hemorrhage in Korean population. Prostaglandins Leukot Essent Fatty Acids. 85, 115-120. doi: 10.1016/j.plefa.2011.07.004

Leiria, L. O., Wang, C. H., Lynes, M. D., Yang, K., Shamsi, F., Sato, M., et al. (2019). 12-Lipoxygenase Regulates Cold Adaptation and Glucose Metabolism by Producing the Omega-3 Lipid 12-HEPE from Brown Fat. Cell Metab. 30, 768.e-783.e. doi: 10.1016/j.cmet.2019.07.001

Liu, Y., Fang, X., Zhang, X., Huang, J., He, J., Peng, L., et al. (2018). Metabolic profiling of murine plasma reveals eicosapentaenoic acid metabolites protecting against endothelial activation and atherosclerosis. Br. J. Pharmacol. 175, 11901204. doi: 10.1111/bph.13971

Nayeem, M. A. (2018). Role of oxylipins in cardiovascular diseases. Acta Pharmacol. Sin. 39, 1142-1154. doi: 10.1038/aps.2018.24

Ostermann, A. I., Willenberg, I., and Schebb, N. H. (2015). Comparison of sample preparation methods for the quantitative analysis of eicosanoids and other oxylipins in plasma by means of LC-MS/MS. Anal. Bioanal. Chem. 407, 1403-1414. doi: 10.1007/s00216-014-8377-8374

Postula, M., Janicki, P. K., Rosiak, M., Eyileten, C., Zaremba, M., Kaplon-Cieslicka, A., et al. (2016). Targeted deep resequencing of ALOX5 and ALOX5AP in patients with diabetes and association of rare variants with leukotriene pathways. Exp. Ther. Med. 12, 415-421. doi: 10.3892/etm.2016.3334

Rahman, S. O., Singh, R. K., Hussain, S., Akhtar, M., and Najmi, A. K. (2019). A novel therapeutic potential of cysteinyl leukotrienes and their receptors modulation in the neurological complications associated with Alzheimer's disease. Eur. J. Pharmacol. 842, 208-220. doi: 10.1016/j.ejphar.2018.10.040 
Robinson, M. D., McCarthy, D. J., and Smyth, G. K. (2010). edgeR: a Bioconductor package for differential expression analysis of digital gene expression data. Bioinformatics 26, 139-140. doi: 10.1093/bioinformatics/btp616

Rosell, A., Vilalta, A., García-Berrocoso, T., Fernández-Cadenas, I., DominguesMontanari, S., Cuadrado, E., et al. (2011). Brain perihematoma genomic profile following spontaneous human intracerebral hemorrhage. PLoS One. 6:e16750. doi: 10.1371 /journal.pone.0016750

Rosso, C., Rosenbaum, D., Pires, C., Cherfils, C., Koujah, N., Mestari, F., et al. (2014). Lipoprotein-associated phospholipase A2 during the hyperacute stage of ischemic and hemorrhagic strokes. J. Stroke Cerebrovasc. Dis. 23, e277-e282. doi: 10.1016/j.jstrokecerebrovasdis.2013.11.024

Sang, M., Wang, X., Zhang, H., Sun, X., Ding, X., Wang, P., et al. (2017). Gene Expression Profile of Peripheral Blood Mononuclear Cells in Response to Intracerebral Hemorrhage. DNA Cell Biol. 36, 647-654. doi: 10.1089/dna.2017. 3650

Shearer, G. C., and Walker, R. E. (2018). An overview of the biologic effects of omega-6 oxylipins in humans. Prostaglandins Leukot Essent Fatty Acids. 137, 26-38. doi: 10.1016/j.plefa.2018.06.005

Shinto, L., Lahna, D., Murchison, C. F., Dodge, H., Hagen, K., David, J., et al. (2020). Oxidized Products of Omega-6 and Omega-3 Long Chain Fatty Acids Are Associated with Increased White Matter Hyperintensity and Poorer Executive Function Performance in a Cohort of Cognitively Normal Hypertensive Older Adults. J. Alzheimers Dis. 74, 65-77. doi: 10.3233/jad- 191197

Sudhahar, V., Shaw, S., and Imig, J. D. (2010). Epoxyeicosatrienoic acid analogs and vascular function. Curr. Med. Chem. 17, 1181-1190. doi: 10.2174/ 092986710790827843

Sun, L., Kang, Q., Pan, Y., Li, N., Wang, X., He, Y., et al. (2019). Serum metabolite profiling of familial adenomatous polyposis using ultra performance liquid chromatography and tandem mass spectrometry. Cancer Biol. Ther. 20, 10171028. doi: 10.1080/15384047.2019.1595277

Walsh, K. B., Zhang, X., Zhu, X., Wohleb, E., Woo, D., Lu, L., et al. (2019). Intracerebral Hemorrhage Induces Inflammatory Gene Expression in Peripheral Blood: Global Transcriptional Profiling in Intracerebral Hemorrhage Patients. DNA Cell Biol. 38, 660-669. doi: 10.1089/dna.2018. 4550

Wang, J., Wang, G., Yi, J., Xu, Y., Duan, S., Li, T., et al. (2017). The effect of monascin on hematoma clearance and edema after intracerebral hemorrhage in rats. Brain Res. Bull. 134, 24-29. doi: 10.1016/j.brainresbull.2017.06.018

Wang, R. X., Chai, Q., Lu, T., and Lee, H. C. (2011). Activation of vascular BK channels by docosahexaenoic acid is dependent on cytochrome P450 epoxygenase activity. Cardiovasc Res. 90, 344-352. doi: 10.1093/cvr/cvq411
Wang, Y., Chen, Q., Tan, Q., Feng, Z., He, Z., Tang, J., et al. (2018). Simvastatin accelerates hematoma resolution after intracerebral hemorrhage in a PPAR $\gamma$-dependent manner. Neuropharmacology 128, 244-254. doi: 10.1016/j. neuropharm.2017.10.021

Willenberg, I., Ostermann, A. I., and Schebb, N. H. (2015). Targeted metabolomics of the arachidonic acid cascade: current state and challenges of LC-MS analysis of oxylipins. Anal. Bioanal. Chem. 407, 2675-2683. doi: 10.1007/s00216-0148369-4

Wójcik, P., Žarković, N., Gęgotek, A., and Skrzydlewska, E. (2020). Involvement of Metabolic Lipid Mediators in the Regulation of Apoptosis. Biomolecules 10:402. doi: 10.3390/biom10030402

Wu, T., Wu, H., Wang, J., and Wang, J. (2011). Expression and cellular localization of cyclooxygenases and prostaglandin E synthases in the hemorrhagic brain. J. Neuroinfl. 8, 22-22. doi: 10.1186/1742-2094-8-22

Xiong, X. Y., and Yang, Q. W. (2015). Rethinking the roles of inflammation in the intracerebral hemorrhage. Transl. Stroke Res. 6, 339-341. doi: 10.1007/s12975015-0402-1

Xu, R., Wang, S., Li, W., Liu, Z., Tang, J., and Tang, X. (2017). Activation of peroxisome proliferator-activated receptor- $\gamma$ by a $12 / 15$-lipoxygenase product of arachidonic acid: a possible neuroprotective effect in the brain after experimental intracerebral hemorrhage. J. Neurosurg. 127, 522-531. doi: 10. 3171/2016.7.JNS1668

Yuan, J. J., Zhang, Q., Gong, C. X., Wang, F. X., Huang, J. C., Yang, G. Q., et al. (2019). Young plasma ameliorates aging-related acute brain injury after intracerebral hemorrhage. Biosci. Rep. 39:BSR20190537. doi: 10.1042/ bsr20190537

Zhao, X., Sun, G., Ting, S. M., Song, S., Zhang, J., Edwards, N. J., et al. (2015). Cleaning up after ICH: the role of $\mathrm{Nrf} 2$ in modulating microglia function and hematoma clearance. J. Neurochem. 133, 144-152. doi: 10.1111/jnc.12974

Conflict of Interest: The authors declare that the research was conducted in the absence of any commercial or financial relationships that could be construed as a potential conflict of interest.

Copyright (c) 2020 Yuan, Chen, Xiong, Zhang, Xie, Huang, Yang, Gong, Qiu, Sang, $\mathrm{Zi}, \mathrm{He}, \mathrm{Xu}$ and Yang. This is an open-access article distributed under the terms of the Creative Commons Attribution License (CC BY). The use, distribution or reproduction in other forums is permitted, provided the original author(s) and the copyright owner(s) are credited and that the original publication in this journal is cited, in accordance with accepted academic practice. No use, distribution or reproduction is permitted which does not comply with these terms. 